\title{
Optically Controlled Coupled Microstripline Microwave Power Attenuator
}

\author{
Raghbendra Singh Tomar,Enakshi K. Sharma and A K Verma \\ (Department of Electronic Science, University of Delhi South Campus, India)7509427936
}

\begin{abstract}
This paper presents an optically controlled coupled microstripline microwave powerattenuator on a high resistivity silicon substrate in the $3 \mathrm{GHz}-7 \mathrm{GHz}$ frequency band. The structure is based on the $10 \mathrm{~dB}$ microstrip directional coupler. Attenuation control is performed by optical control of the open end resistances. The open end act as a variable resistance under sufficient optical illumination conditions. The attenuator has been designed for $10 \mathrm{~dB}$ and $25 \mathrm{~dB}$ attenuation control for one-port and two port optical illuminations respectively. The analysis has been carried out for one port illumination using a 30mW, 850nm laser diode and two ports illumination using two $20 \mathrm{~mW}$, $650 \mathrm{~nm}$ laser diode. The attenuator has been modeled by the Computer Simulation Technique's Micro Wave Studio (CST MWS) 3D EM simulator. At 5GHz, the structure provides a continuous variation of $S 41$ between 0 and $25 \mathrm{~dB}$ with both open end illumination.
\end{abstract}

Keywords: Attenuator, Coupled Lines, CST MWS, Directional Coupler, Optical Control

\section{Introduction}

In the last years, there has been a great interest in the development of laser controlled systems because of the fact that the optical control of microwave devices offers high isolation between the controlling optical beam and the controlled microwave signal, short response time, high-power handling capacity, immunity to electromagnetic interference and low cost [1]. Optical control of microwave devices based on the photoconductivity effect. When a semiconductor is illuminated with a photon of the appropriate wavelength,an electron-hole pair is generated in the semiconductor substrate creating a variable load that changes the propagation characteristics in the microwave device. This change in characteristics has applications such as in tunable filters [2], phase shifters [3], and microwave matching techniques [4]. One of the new promising applications of microstrip technology is the optically controlled microwaveattenuators [5].Such optical control is based on the fact that when photons of energy greater than the band gap are incident on the silicon substrate, electron-hole plasma created by light absorption [6]. The optically generated plasma at the end of an open microstripforms an optically controlled load [7-8]. The main reason of using these devices is due to the demand of new and emerging applications, which leads to the development of circuits and subsystems in the optical range. In this paper, a modified directional coupler with two wide open-ended coupled lines is presented. The even-odd mode technique [9-10] is employed to analyze and synthesize such a structure. After that, the laser tuning is achieved by modifying the electrical length of the open-ended coupled lines. An optically controlled microwave attenuator using a microstrip directional coupler on high resistivity silicon substrate has been experimentally demonstrated by Haider et al. [11]. They used two ports illumination by a high power argon laser $(600 \mathrm{~mW}, 514 \mathrm{~nm})$ to obtain $10 \mathrm{~dB}$ attenuation control at $6 \mathrm{GHz}$ in the frequency band 3-8GHz. However, neither an analytical nor a simulated model has been presented by them. In this paper we present investigations on oneport and two-port illuminated coupled microstrip attenuators on the high resistivity silicon substrate, in the frequency band $3-7 \mathrm{GHz}$. One port illumination using a $30 \mathrm{~mW}, 850 \mathrm{~nm}$ laser diode and two ports illumination using two $20 \mathrm{~mW}, 650 \mathrm{~nm}$ laser diode has been done. The attenuator has been modeled by the Computer Simulation Technique's Micro Wave Studio (CST MWS) 3D EM simulator [12], taking into account the SMA to microstrip transition.

\section{Theory Of Optically Controlled Attenuator}

The optical control of microstripattenuators based on the phenomenon of optical load discussed in [78]. When the open end on the microstrip line is illuminated by a laser spot, electron-hole pairs are created by light absorption spread into the substrate due to carrier diffusion to give an inhomogeneous carrier distribution in the illuminated substrate. The absorption and penetration depth of the plasma depend on the optical wavelength and substrate parameters. Such electron-hole plasma created at the end of the open microstrip line due to illumination by laser spot changes the dielectric constant within the illuminated region in the semiconducting substrate. The optically illuminated region can be considered as a cylinder filled with a dielectric constant of complex dielectric constant whose value varies with substrate depth and can be modeled as a capacitor with a lossy dielectric, which gives a complex capacitance. This leads to an equivalent circuit model 
of a capacitance in parallel to a resistance. The resistance decreases from several kilo-ohms in dark state to a few ohms with increase in optical intensity. Considering a uniform distribution of the photo induced charges, the relative complex dielectric constant in the microwave range can be written as

$\varepsilon_{r}^{*}=\varepsilon^{\prime}-j \varepsilon^{\prime \prime}$

The real and imaginary part of the complex dielectric constant given by

$\varepsilon^{\prime}=\varepsilon_{L}-\frac{e^{2} \tau_{p}{ }^{2}\left(\frac{p_{l}}{m_{p l}^{*}}+\frac{p_{h}}{m_{p h}^{*}}\right)}{\varepsilon_{0}\left(1+\omega^{2} \tau_{p}^{2}\right)}+\frac{n_{e} e^{2} \tau_{e}^{2}}{m_{e}^{*} \varepsilon_{0}\left(1+\omega^{2} \tau_{e}^{2}\right)}$

$\varepsilon^{\prime \prime}=\frac{e^{2} \tau_{p}\left(\frac{p_{l}}{m_{p l}^{*}}+\frac{p_{h}}{m_{p h}^{*}}\right)}{\varepsilon_{0} \omega\left(1+\omega^{2} \tau_{p}^{2}\right)}+\frac{n_{e}{ }^{2} e^{2} \tau_{e}}{m_{e}{ }^{*} \varepsilon_{0} \omega\left(1+\omega^{2} \tau_{e}^{2}\right)}$

Where $\varepsilon_{\mathrm{L}}$ is the relative dielectric constant in the dark state, e is electronic charge, $\tau_{\mathrm{p}}$ and $\tau_{\mathrm{e}}$ are the collision time for electron and hole, $p_{l}$ and $p_{h}$ are the densities of light and heavy holes, $m_{p l}$ and $m_{p h}$ are the effective mass of the light and heavy holes, respectively, $v$ is the microwave pulsation.The complex refractive indexis expressed as follows [8]

The corresponding complex refractive index $n^{*}$ of illuminated silicon can be defined as

$n^{*}=\eta-j \kappa$

where, $\eta$ and $\kappa$ are the real and imaginary part of the refractive index, respectively, and are related to $\varepsilon_{r}^{*}$ by the relation

$n^{* 2}=\varepsilon_{r}^{*}$

complex propagation constant $\beta^{*}$ defined as

$$
\begin{aligned}
\beta^{*}=\beta-j \alpha= & k_{0} \sqrt{\varepsilon^{\prime}-\mathrm{j} \varepsilon^{\prime \prime}} \\
& =k_{0}(\eta+j \kappa)
\end{aligned}
$$

The real part $\beta$ is phase constant and imaginary part $\alpha$, the attenuation constant of the complex propagation constant of a wave. The quantity $\beta / k_{0}$ referred to as the slowing factor, defines the ratio of phase velocity in free space to phase velocity in the semiconductor

$\delta$ referred to skin depth and defined as

$\delta=\frac{1}{\alpha}$

$\beta / k_{0}$ and $\alpha$ increases as carrier concentration or frequency increases while, the skin depth $\delta$ decreases with either carrier concentration or frequency. [10]. If the conductivity becomes too large, the depth of the conducting region will be defined by the skin depth and not by the diffusion length. From the value of $\beta^{*}$, it can be seen that the presence of the plasma region alters the wave velocity. Another issue is that $\alpha$ increases together with $\beta^{*}$.This will obviously reduce the transmission coefficient.If the diffusion length is small compared to the absorption depth $1 / \alpha$ the conductivity and resistance becomes 


$$
\begin{aligned}
& \sigma(y)=(1-R) e\left(\mu_{n}+\mu_{p}\right) \frac{S \lambda_{p} \tau}{h c}\left(\frac{P}{A}\right) e^{-\alpha y} \\
& R=\frac{h c}{(1-R) e\left(\mu_{n}+\mu_{p}\right) S \lambda_{p} \tau P}\left(e^{\alpha d}-1\right)
\end{aligned}
$$

In this case the optically induced resistance is controlled by radiation absorption characteristics of semiconducting substrate and radiation wavelength. The relation between optical power and optically induced resistance is given in table 1 .

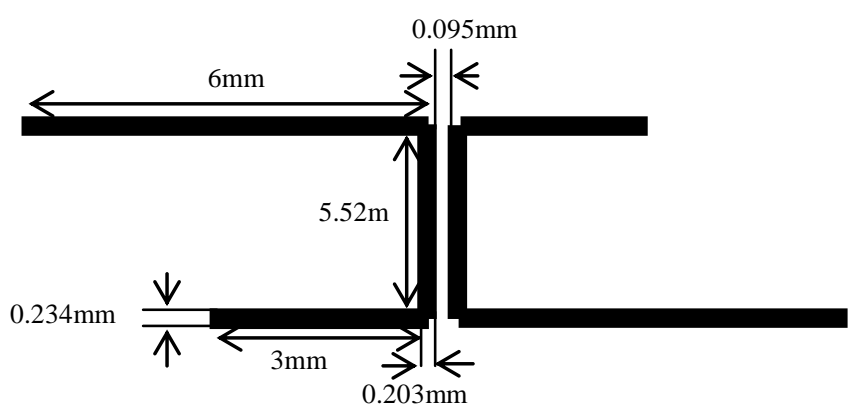

Figure:1Optically Controlled Coupled Microstrip line Microwave Power Attenuator

TABLE: 1 the relation between optical power and optically induced resistance

\begin{tabular}{cc}
\hline Power $(\mathrm{mW})$ & Resistance $(\Omega)$ \\
\hline 5 & 280.38 \\
10 & 140.19 \\
15 & 93.46 \\
20 & 70.09 \\
25 & 56.07 \\
30 & 46.73 \\
35 & 40.05 \\
40 & 35.04 \\
\hline
\end{tabular}

\section{Design and Simulation}

Fig. 1 shows the layout of the proposed attenuator. The primary structure is quarter wavelength $10 \mathrm{~dB}$ directional coupler at the center frequency of $5 \mathrm{GHz}$. The coupler consists of two conductor layers interleaved by one substrate between the conductor layers. The coupled microstrip lines designed on the high resistivity silicon substrate having dielectric constant 11.8 and resistivity $3000 \Omega-\mathrm{cm}$ and thickness $285 \mu \mathrm{m}$. The dimensions of the coupled lines are calculated from CAD oriented software called microwave office TCAD [13] using the even and odd mode impedances. In fig. 1, for the illumination the port 2 and port 3 left open. The port- 1 is the input port and the isolated port- 4 is the output port of the attenuator. In this configuration the four port device becomes a new two port device. Due to mismatch at the coupled port-2, the microwave signal gets reflected and appears at the output port-4. This reflection is a maximum under dark condition. With optical illumination at port-2, the load resistance reduces at port-2, which in turn reduces the power at the output port- 4 . The minimum available output power at the port- 4 is determined by the directivity of the mictrostrip coupler. A large reflection occurs on the main line resulting into poor return loss because port-3 is open. In the fig. 2 above described phenomena can be seen. In fig. 2a when the device is four ports coupler maximum surface current density is on the through port 3 and a coupling of $10 \mathrm{~dB}$ power is showing on port 2 . In fig. $2 \mathrm{~b}$ when port 2 and 3 keep open the 10dB down current density is on output port 4 . S11 can be improved by optical illumination of port-3, as the illumination creates a better optically controlled matched load. At the center frequency of the coupler, we can estimate $\mathrm{S} 11$ and $\mathrm{S} 14$ for a coupling coefficient $\mathrm{C}$ by the following expressions: 
$\mathrm{S}_{11}=2 \mathrm{C}^{2}-1$

$\mathrm{S}_{14}=2 \mathrm{C} \sqrt{\left(1-\mathrm{C}^{2}\right)}$

The propagation characteristics are calculated by the three-dimensional (3D) simulator CST Microwave Studio [12], based on the finite integration technique. Themicrostrip to coaxial transition produces discontinuities which in turn creates open end losses and non smoothness in response of S41 due to mismatch. The fig. 3 is the modeling of transition from SMA to microstrip. The SMA connector has been properly designedand modeled by us with LC network. This modeling provides smoother response for S14.

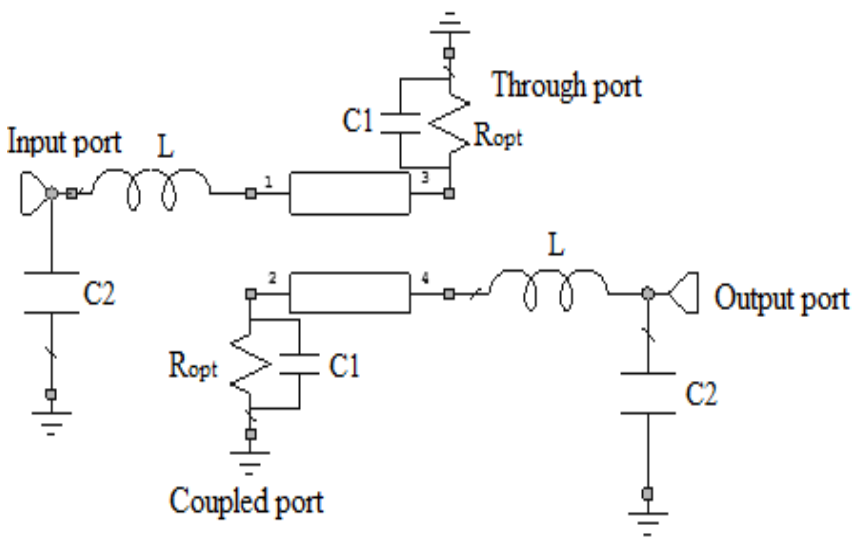

Figure:2Equivalent Circuit ofOptically Controlled Coupled Microstrip line Microwave Power Attenuator with the SMA to microstrip transition.

We can obtain optical control of the attenuator either by one-port or by two-port illumination.In the case of one-port illumination either of the two ports, port-2 or port-3, can be illuminatedwhile the other is open or terminated in a $50 \Omega$ matched load Fig. 3 shows, modeling of the SMA to microstrip line transition at both the input port- 1 and atthe output port- 4 by the LC-network. The values of $\mathrm{C} 1, \mathrm{C} 2$ and $\mathrm{L}$ are $0.1 \mathrm{pF}, 0.3 \mathrm{pF}$ and $1.8 \mathrm{nHrespectively.}$

Both open ends have been simulated by a parallel RCload with R changing according to illumination level at port-2. The value of $\mathrm{C}$ has beenestimated by the open end discontinuity. The CST MWS simulated response of the attenuator with these terminations (Fig. 4) show that under the dark condition maximum S41is $0 \mathrm{~dB}$ at $4.5 \mathrm{GHz}$ and comes down to $-8 \mathrm{~dB}$, i.e., $8 \mathrm{~dB}$ attenuation control for the load change from $5000 \Omega$ to $50 \Omega$. The attenuation control is $10 \mathrm{~dB}$ when load comes down to $30 \Omega$. Moreover,modeling of transition by LC network disturbs the smoothness of response for S41. Onillumination of both the through and coupled ports, attenuation control more than $20 \mathrm{~dB}$ couldbe obtained

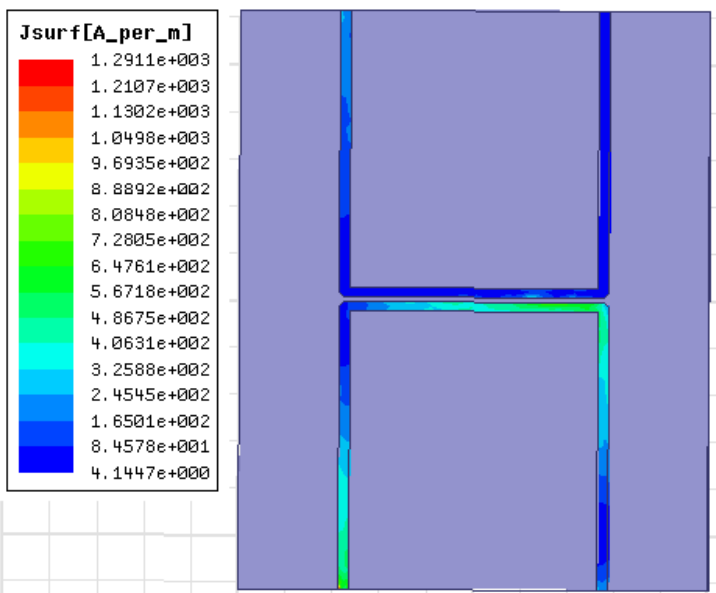

(a)

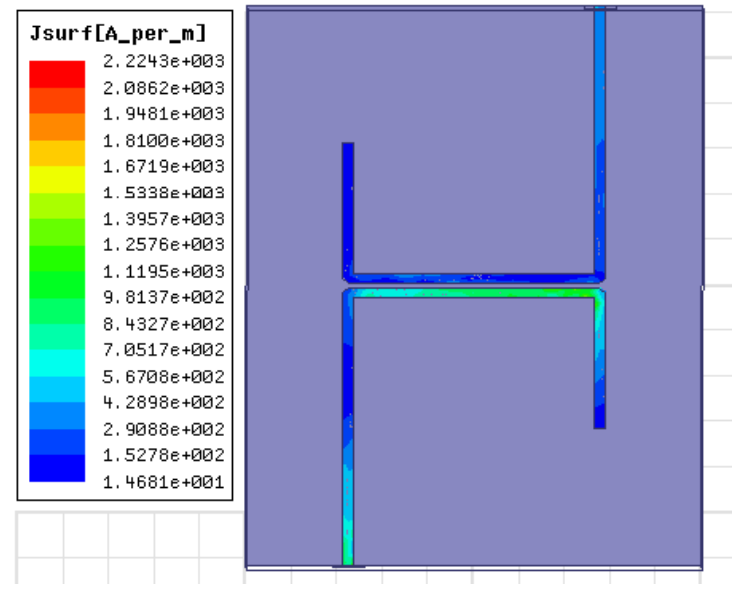

(b)

Figure:3 Current density distribution in (a) primary directional coupler and in (b) optically controlled attenuator when both port open (maximum power at port four) 


\section{Results And Discussion}

Fig. 4 and fig. 5 show the CST MWS simulatedtransmission responsesfor attenuator with illumination at port- 2 and port-3 open. A changing optical resistance has been used at the place of optical illumination. The S21 of the transmitted wave depends strongly on the injected optical power. The magnitude of S21 not remains practically constant at one given frequency.The variable optical control has been obtained by changingresistances $30 \Omega, 50 \Omega, 100 \Omega, 500 \Omega$, and $5000 \Omega$, where $30 \Omega$ resistancesmeans $20 \mathrm{~mW}$ power and $5000 \Omega$ resistance corresponds to the dark condition. The nature of S41 and S11 showing the correctness of the attenuatormodel. The simulated results for $\mathrm{S} 21$ show two maxima, at $4.5 \mathrm{GHz}$ and $8 \mathrm{GHz}$, with a dip at $7 \mathrm{GHz}$. The S11 for port 2 illuminations gives dip at 4.6GH and at 8GHz. Fig. 6 and fig. 7 show theport 3 illumination simulated results. 221 show the same pattern that is maxima at $4.5 \mathrm{GHz}$ and $8 \mathrm{GHz}$, with a dip at $7 \mathrm{GHz}$. But for the S11 it changes and gives vary low reflection for $500 \Omega$ resistance. For one port illumination whether it is at port 2 or at port 3 , the simulation results show attenuation control of $10 \mathrm{~dB}$ from the dark condition load resistance $5000 \Omega$ to the illuminated condition load resistance $30 \Omega$. Fig. 8 and fig. 9 show results for both port- 2 and port- 3 are illuminated simultaneously by two independent $20 \mathrm{~mW}, 650 \mathrm{~nm}$ laser diodes. The Fig. 8 shows attenuation control of $25 \mathrm{~dB}$ and fig. 9 shows that S11 degrades with increase in illumination. The nonsmoothness in response of S41 is due to the transition from SMA to microstrip which has been modeled, by us with LC network. However, a properly designed transition can provide smoother response for S41. An attenuator designed for $6 \mathrm{~dB}$ coupling coefficient will provide better return loss. Thus, for a $10 \mathrm{~dB}$ coupler $\mathrm{S} 11$ is $-1.94 \mathrm{~dB}$ and $\mathrm{S} 41$ is $-4.4 \mathrm{~dB}$, whereas, for a $6-\mathrm{dB}$ coupler S11 improves to $-6.06 \mathrm{~dB}$ and $\mathrm{S} 14$ is $-12.35 \mathrm{~dB}$. Haider et al. [6] used a $6 \mathrm{~dB}$ coupling co-efficient for their design. However, for ease in fabrication we used a $10 \mathrm{~dB}$ coupling co-efficient in our design of the optically controlled attenuator. With optical illumination at port-2, the load resistance reduces at port-2, which in turn reduces the power at the output port-4. The minimum available output power at the port- 4 is determined by the directivity of the mictrostrip coupler. A large reflection occurs on the main line resulting into poor return loss because port-3 is open. S11 In fig. 5 the increasing optical illumination hasbeen simulated by a decrease in load variation from $5000 \Omega$ (dark condition) to $50 \Omega$. However,the return loss is not satisfactory. It can be improved upto $20 \mathrm{~dB}$ for the case of illumination atport-2 by terminating port-3 in a $50 \Omega$ load with simultaneous improvement in attenuation control (10dB). The simulated results for the case of illuminated port-2 with port- 3 open, showthat the maximum coupling frequency shifts from $4.5 \mathrm{GHz}$ to $4.6 \mathrm{GHz}$.

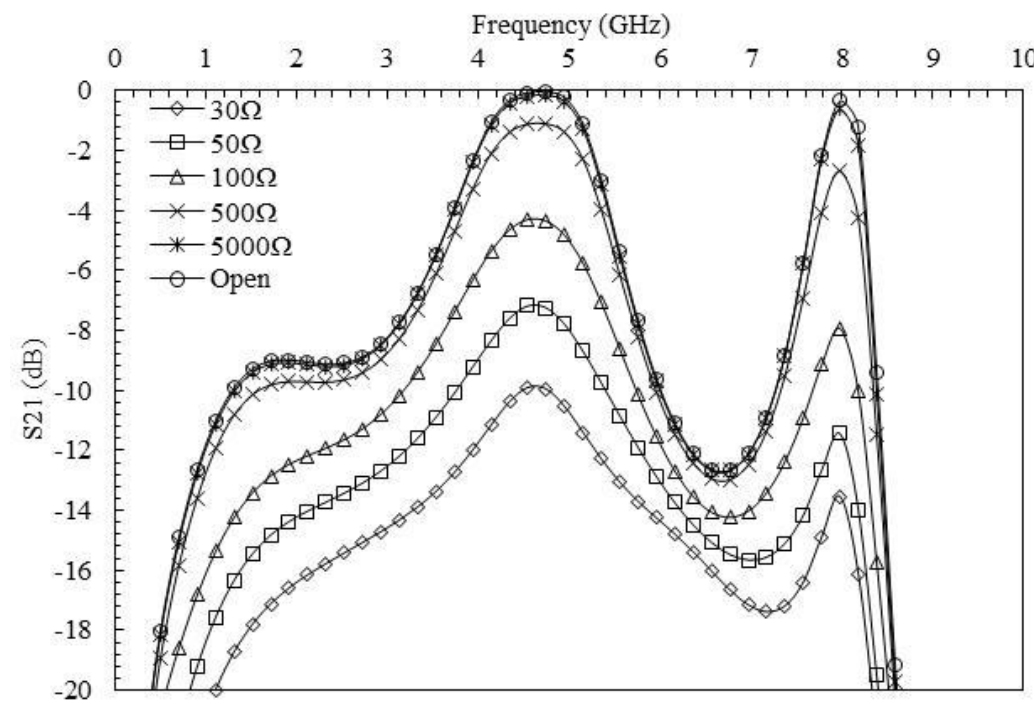

Figure:4 Variation of S21 when port 2 terminated with optical load and port 3 remain open 


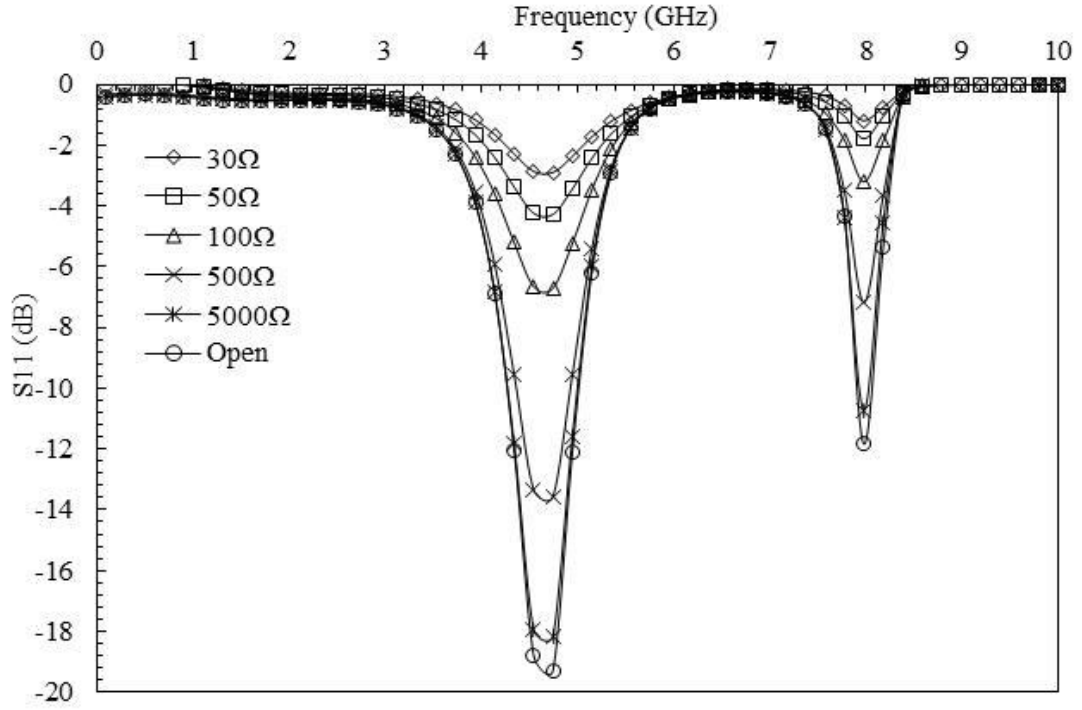

Figure:5 Variation of S11 when port 2 terminated with optical load and port 3 remain open

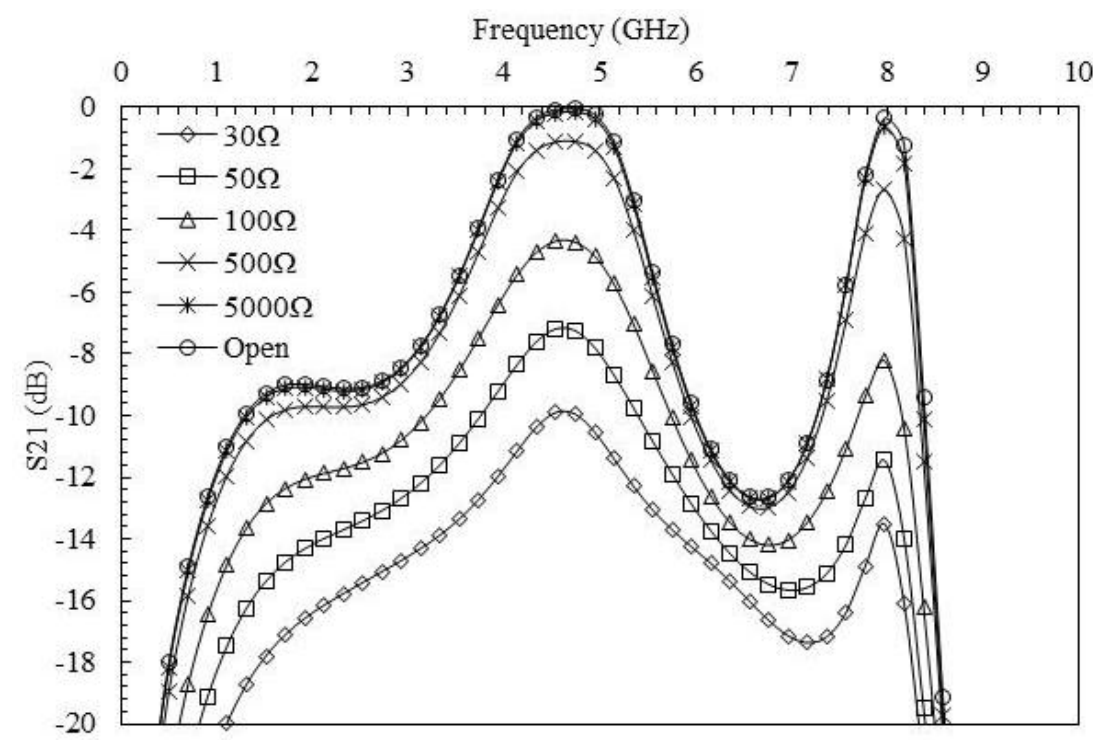

Figure:6 Variation of S21 when port 3 terminated with optical load and port 2 remain open

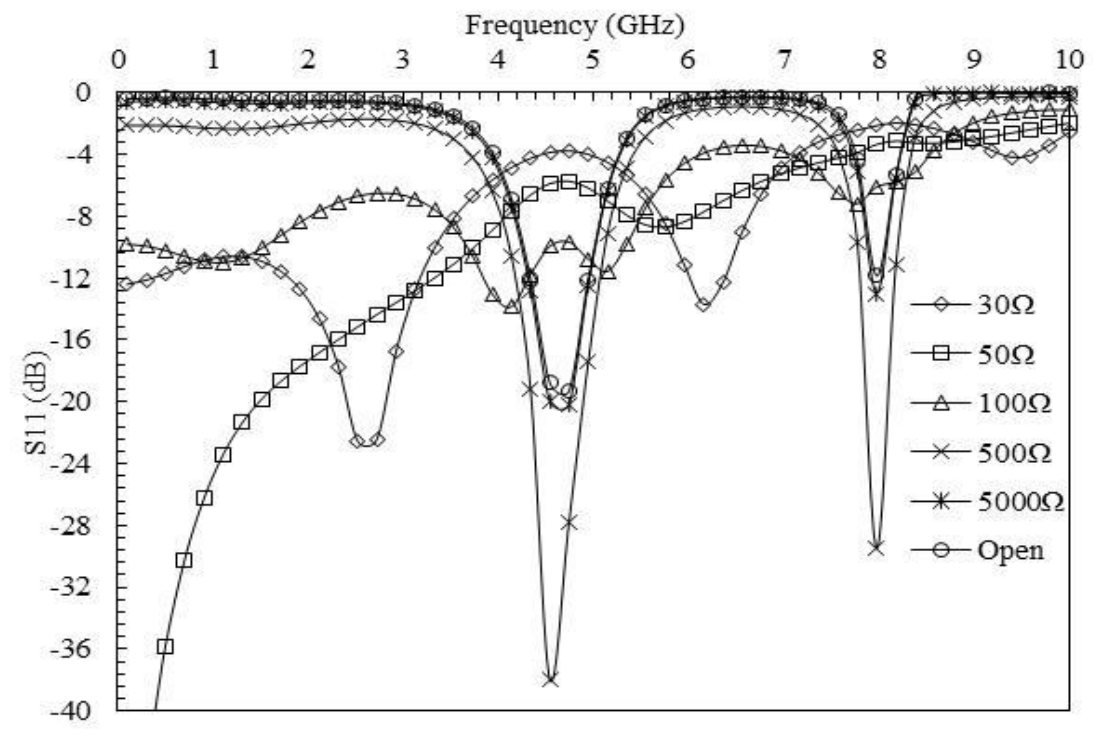

Figure:7 Variation of S21 when port 3 terminated with optical load and port 2 remain open 


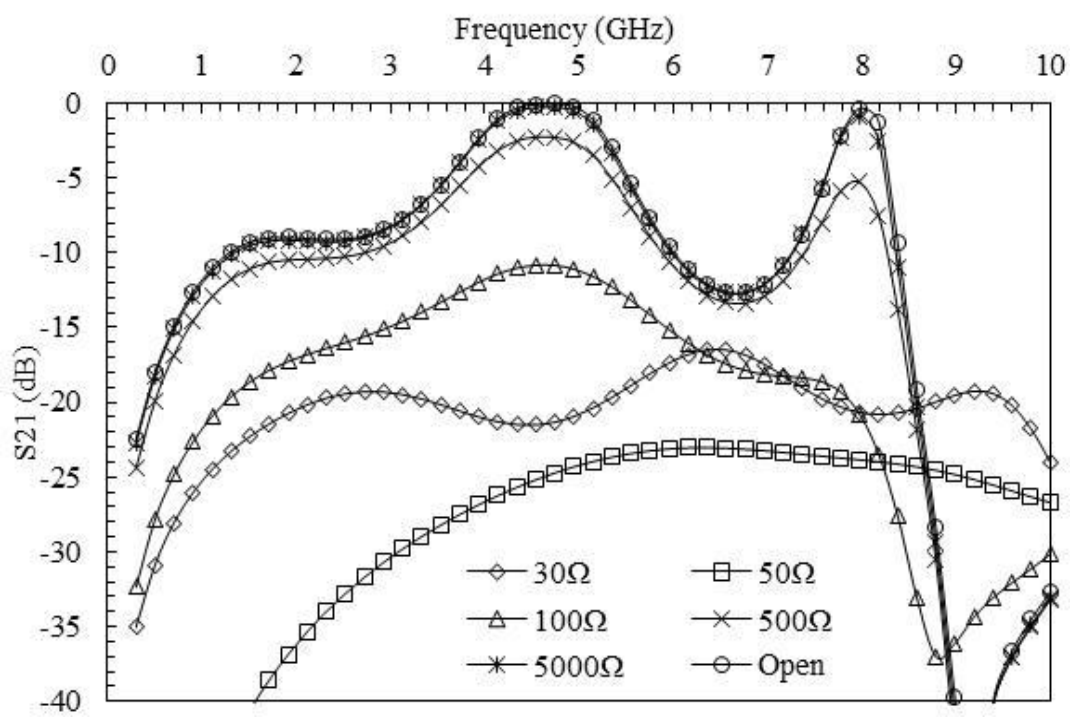

Figure:8 Variation of S21 when both port 2 and port 3 terminated with optical load Frequency $(\mathrm{GHz})$

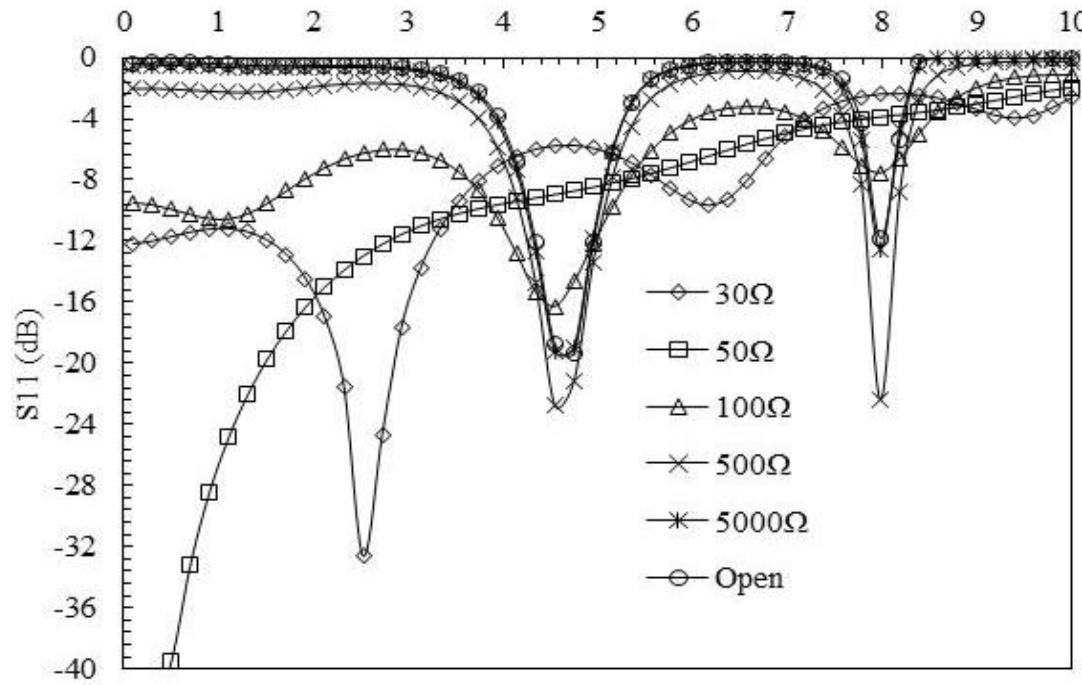

Figure:9 Variation of S21 when both port 2 and port 3 terminated with optical load

\section{Conclusion}

An optical control of $10 \mathrm{~dB}$ and $20 \mathrm{~dB}$ attenuation could be obtained by the one and two ports illumination respectively. In case of the one port illumination, the return loss could be improved by terminating the port- 2 in the $50 \Omega$ matched load. In case of two ports illumination, return loss could be improved by designing the attenuator for $6 \mathrm{~dB}$ coupling co-efficient. At $650 \mathrm{~nm}$ illumination, optical load is modeled by a resistance. The transition from SMA to microstip is modeled by LC network and CST MWS simulated results correctly predict behavior of the optically controlled attenuator.

\section{Acknowledgements}

For the financial support the CSIR-India is acknowledged.

\section{References}

[1] Herczfeld, P.R., Daryoush, A.S., Contarino, V.M., Rosen, A., Turski, Z., and Khana, A.P.S.: 'Optically controlled microwave devices and circuits.' IEEE MTT-S Int. Microw. Symp. Dig., 1985, pp. 211-214

[2] Platte, W.: 'Periodic-structure photoexcitation of a silicon coplanar wave guide for selective optoelectronic microwave control', IEEE Trans. Microw. Theory Tech., 1990, 38, (5), pp. 638-646

[3] Cheung, P., Neikirl, D., and Itoh, T.: 'Optically controlled coplanar waveguide phase-shifter', IEEE Trans. Microw. Theory Tech., 1990, 38, pp. 586-594

[4] Safwat, A., Khalil, D., Elhennawy, H., and Ragaie, H.: 'Quasi-static analysis of an optically illuminated directional coupler', IEEE Trans. Microw. Theory Tech., 1997, 45, pp. 1351-1357 
[5] Lee, S., Kuga, Y., and Mullen, R.A.: 'Optically tunable, millimeter-wave attenuator based on layered structures', Microw. Opt. Technol. Lett., 2000, 27, (1), pp. 9-13

[6] J. Haidar, A. Vilcot, M. Bouthinon and E. Pic., "Optically controlled passive microwavestructures", Sino-French Workshop on Fibre\& Integrated Optics, Sept. 19-21, Shanghai.

[7] B. Boyer, J. Haidar,A. Vilcot and M. Bouthinon," Tunable microwave load based on biasedphotoinduced plasma in silicon', IEEE Trans. Microwave Theory Tech., Vol. 45, pp. 1362-1367.Aug, 1997.s

[8] A.K. Verma, Enakshi K. Sharma, Nasimuddin, A. Bhaduria and B. R. Sigh, "Opticallycontrolledmicrostrip load", National Sym. On Advances in Microwaves and Light waves, N.Delhi, India, Mar., 2000, pp.250-254.

[9] D. M. Pozar, Microwave Engineering, 3rd ed., New York: Wiley,2005; pp337-345. ISBN 0-471-44878-8.

[10] R. Mongia, I. Bahl, P Bharia, RF and Microwave Coupled-LineCircuits, Norwood MA, Artech House, 1999, pp. 4-13. ISBN0-89006830

[11] Jihad Haidar, Anne Vilcot and Michel Bouthinon, 'Optically tunable microwave attenuator using a quarter-wave microstrip coupler' Microwave and Optical Technology Letters, Volume 10, Issue 6, pages 313-314, 20 December 1995

[12] User manual CST Microwave Studio, 2013

[13] User manual AWR Microwave office, 2002 